\title{
Refração ocular por retinoscopia em faixa em cães da raça Fila Brasileiro*
}

\section{Ocular refraction by streak retinoscopy in the canine Fila Brasileiro breed}

\author{
Ana Maria Barros Soares, ${ }^{* \star},{ }^{* * *}$ José Luiz Laus, ${ }^{* * *}$ Jacqueline Provenzano, ${ }^{* \star *}$ Bernadete Ayeres, ${ }^{* \star *}$ Priscila de \\ Marssilac, ${ }^{* * * *}$ Cristiane Millward, ${ }^{* * * *}$ Paula Diniz Galera, ${ }^{* * *},{ }^{* * * * *}$
}

\begin{abstract}
Resumo
Realizou-se a refração objetiva de 104 olhos de 52 cães adultos da raça Fila Brasileiro. Todos os animais mostraram grau leve de hipermetropia, com média de 1,12 $\pm 0,41$ dioptrias (D). Identificou-se baixo grau de astigmatismo hipermetrópico composto de $0,50 \mathrm{D}$ em $16,35 \%$ dos olhos. Anisometropia hipermetrópica composta de 0,50 D constituiu-se achado freqüente, e foi considerada normal.
\end{abstract}

Palavras-chave: esquiascopia, cão, olho.

\begin{abstract}
Streak retinoscopy, was performed in 104 of 52 adult Fila Brasileiro breed dogs. All animals showed low-grade hypermetropia, with mean average of $+1.12 \pm 0.41 \mathrm{D}$. Slightly degree of compound hypermetropic astigmatism $(0,50 \mathrm{D})$ was found in $16.35 \%$ of the eyes. Compound hypermetropic anisometropia of $0.50 \mathrm{D}$ was frequently observed and was considered normal.
\end{abstract}

Keywords: retinoscopy, dog, eye.

\section{Introdução}

Pesquisas visando ao desenvolvimento de técnicas para correção de ametropia em animais constitui campo pouco explorado em Veterinária (Ofri, 1999). A inclusão e a associação de técnicas para a avaliação da refração ocular são recomendadas quando da triagem e seleção de animais concebidos para funções específicas (Murphy et al., 1997), assim como para a correção de ametropias em animais fácicos ou afácicos (Davidson et al., 1993; Rosolen et al., 1995; Murphy et al., 1997; Ofri, 1999; Strubbe e Gelatt, 1999).

A esquiascopia ou retinoscopia com luz em faixa é técnica de determinação do poder refrativo ou estado dióptrico do olho (Davidson, 1997; Strubbe e Gelatt, 1999). Constitui-se no mais exato dos métodos de determinação da refração estática ocular (Alves, 1999; Alves e Avakian, 2000; Netto, 2000) e o mais utilizado em Veterinária (Strubbe e Gelatt, 1999). A técnica permite a avaliação de estados de afacia e estudos de implantes de lentes intra-oculares (Pollet, 1982; Gaiddon et al., 1991; Davidson et al., 1993; Gaiddon et al., 1996; Gaiddon et al., 2000), bem como a determinação do estado refracional em diferentes espécies e raças (Murphy et al., 1992; Gaiddon et al., 1996; Gilger et al., 1998; Mutti et al., 1999).
A retinoscopia em faixa consiste em se observar o movimento do reflexo do feixe luminoso através da pupila (Alves, 1999; Netto, 2000). O aparelho congrega dois sistemas, um de observação e outro de iluminação. O retinoscópio projeta uma faixa de luz linear de vergência ajustável, que é refletida a partir da retina do olho examinado, atravessa os componentes ópticos do bulbo, passa pelo retinoscópio, sendo observada pelo examinador na abertura pupilar do paciente. Da visibilização da emergência desses raios, determina-se o erro refracional (Alves, 1999; Alves e Avakian, 2000).

O escurecimento da sala é, em geral, o suficiente para se obter abertura pupilar adequada à realização da retinoscopia. Devido à limitada habilidade de acomodação da lente dos cães 1,00 a 3,00 D (Miller e Murphy, 1995), estudos demonstraram que a indução da cicloplegia não é imprescindível ao exame (Nowak e Neumann, 1987; Murphy et al., 1992). Cabe ressaltar que a indução de midríase completa pode gerar reflexos irregulares, prejudicando a identificação de neutralidade (Davidson, 1997; Alves e Avakian, 2000), em face da exposição da área periférica da córnea e da lente que, geralmente, exibem refração diferente da área axial (Alves e Avakian, 2000).

\footnotetext{
* Parte de tese de doutorado do Programa de Pós-Graduação em Medicina Veterinária, área de Concentração em Cínica Médica Veterinária da Universidade Estadual Paulista (UNESP - Jabiticabal). Apoio CAPES - PICDT/Universidade Federal Fluminense.

** Universidade Federal Fluminense. Niterói, RJ, Brasil. soaresa@vm.uff.br

*** Universidade Estadual Paulista. Jaboticabal, SP, Brasil.

**** Oculistas Associados. Rio de Janeiro, RJ, Brasil.

***** Veterinária autônoma. RJ, Brasil.

****** União Pioneira de Integração Social/UPIS - Brasília, DF
} 
Em ambiente semi-escuro (Nowak e Neumann, 1987; Strubbe e Gelatt, 1999), deve-se manter a cabeça do paciente estáti$\mathrm{ca}$, procurando-se alinhar o seu olhar com o do examinador (Gaiddon et al., 1996; Davidson, 1997). O alinhamento óptico é indispensável para a obtenção de resultados precisos, o que torna o exame desafiador, em virtude da constante movimentação do paciente. Em Veterinária, assim como em oftalmologia pediátrica, a retinoscopia empreita a cooperação do paciente e exige destreza do examinador (Davidson, 1997).

A contenção mecânica, com a estabilização da cabeça constitui-se, então, em procedimento factível (Nowak e Neumann, 1987). Sedação ou anestesia podem ser consideradas, se necessárias. Não obstante, há drogas potencialmente capazes de gerar resultados não fidedignos ou de limitar a execução do procedimento, por promoverem alterações como mudança do volume ocular, nistagmo, protrusão da terceira pálpebra e miose (Davidson, 1997).

No curso da retinoscopia, o retinoscópio é posicionado a uma distância conhecida de 50 ou 66 centímetros do olho do paciente (Davidson, 1997; Alves e Avakian, 2000). O intervalo de 66 centímetros é o mais empregado, pois minimiza erros, facilita a consecução de cálculos matemáticos e é, normalmente, a distância facilmente alcançada pelo braço da maioria dos indivíduos (Alves e Avakian, 2000).

Com o retinoscópio mantido a $66 \mathrm{~cm}$ do olho do paciente (Davidson, 1997; Alves e Avakian, 2000), ajusta-se a vergência ao efeito do espelho plano. Alinhando-se as imagens de Purkinje da superfície anterior da córnea e da lente, obtém-se o alinhamento óptico que garante ao examinador projetar os raios de luz incidentes no eixo óptico do olho. $\mathrm{O}$ examinador deve, então, movimentar a cabeça suavemente ou movimentar o instrumento gentilmente, de modo a poder fazer a varredura, através da pupila do paciente, perpendicular ao eixo do feixe. O poder dióptrico aferido corresponde ao do meridiano ocular na direção do movimento e não na direção do feixe de luz (Davidson, 1997).

Varreduras devem ser feitas nos meridianos oculares vertical e horizontal (Pollet, 1982; Davidson, 1997). O movimento oscilatório do aparelho permitirá que se observe a faixa luminosa na abertura pupilar. $\mathrm{O}$ reflexo luminoso irá se mover na mesma direção do movimento oscilatório do retinoscópio, ou em direção oposta, dependendo do erro de refração do paciente. Quando o reflexo exibir movimento "a favor", conclui-se por um meridiano hipermetrope, emetrope ou míope menor que $-1,50 \mathrm{D}$. De outra forma, se o reflexo exibir movimento "ao contrário", conclui-se por um meridiano míope maior que 1,50 D (Alves e Avakian, 2000).

Com a retinoscopia, objetiva-se encontrar o ponto remoto do olho míope ou o ponto de neutralização (Alves e Avakian, 2000). A neutralização é caracterizada por um reflexo que preenche, completamente, a pupila sem que se tenha movimento em qualquer direção (Nowak e Neumann, 1987; Strubbe e Gelatt, 1999). Na vigência de movimentação do reflexo luminoso, lentes acessórias de poder dióptrico conhecido são interpostas entre o examinador e o paciente, no escopo de se determinar o grau de refração (Alves e Avakian, 2000). As lentes mais utilizadas são as barras de esquiascopia posicionadas a 1,00 ou 2,00 centímetros da córnea do paciente (Davidson, 1997). Na ocorrência de movimento "a favor", devem ser adicionadas lentes positivas; já quando ele se dá "ao contrário", adicionam-se as negativas. Do poder dióptrico obtido no ponto de neutralização subtrai-se o valor dióptrico da distância de trabalho (Alves e Avakian, 2000). Em um olho emetrope, com a retinoscopia realizada a uma distância de trabalho de $66 \mathrm{~cm}$, necessita-se de lente de $+1,50 \mathrm{D}$ para que se atinja a neutralização. Condicionadas estas variáveis, determina-se o poder dióptrico total obtido na neutralização e subtrai-se $1,50 \mathrm{D}$ relativo ao valor dióptrico dessa distância de trabalho (Strubbe e Gelatt, 1999; Alves e Avakian, 2000). Na ausência de astigmatismo, a retinoscopia mostrará o mesmo ponto de neutralização em qualquer dos meridianos. Em olhos astigmatas, os pontos de neutralização mostram diferentes valores equivalendo-se o poder dióptrico a diferença entre eles (Alves e Avakian, 2000).

A maioria dos cães fácicos apresenta de 0,50 a 1,00 D de emetropia (Pollet, 1982; Nowak e Neumann, 1987; Neumann, 1988; Murphy et al., 1992; Nelson et al., 1996, Davidson, 1997). Encontram-se estudos que falam de populações com tendência à miopia (Pollet, 1982; Nowak e Neumann, 1987; Gaiddon et al, 1996; Nelson et al., 1996; Mutti et al., 1999) e outros que inferem a hipermetropia (Wyman e Donavan, 1965; Murphy et al., 1992).

Sabe-se que raça, porte, habitat, predisposição familiar e idade podem estar relacionados com erros de refração (Murphy et al., 1992; Davidson et al., 1993; Nelson et al., 1996; Gaiddon et al., 1996; Mutti et al., 1999).

Estudos demonstraram que algumas raças tendem a apresentar miopia tais como: Pastor Alemão, Rottweiler, Schnauzer Miniatura, Poodles Toy e Miniatura (Murphy et al., 1992; Mutti et al., 1999), Retriever do Labrador (Nelson et al., 1996; Mutti et al., 1999), Cocker Spaniel Americano, Dogue Alemão, Pug, Samoieda, Elkhound Norueguês, Papillon, Schipperke, Pastor de Shetland, Terriers, Colie Pelo Longo e entre Teckel Miniatura. Hiperopia foi constatada em exemplares das raças Pastor Alemão, Shar-pei (Murphy et al., 1992), Malamute do Alasca, Pastor Australiano, Basenji, Pastor Belga, Pastor de Berna, Border Colie, Cheasapeake Bay Retriever, Clumber Spaniel, Doberman, Springer Spaniel Inglês, Field Spaniel, Golden Retriever, Husky Siberiano (Mutti et al., 1999). Em animais idosos a esclerose senil favorece o aparecimento da miopia (Murphy et al., 1992).

Gaiddon et al. (1996) não encontraram qualquer correlação entre o tipo de crânio e erros de refração. Todavia, cães de pequeno e médio porte mostraram tendência à miopia, enquanto os de grande porte, à hipermetropia. Os autores observaram, ainda, que o habitat influenciava no erro de refração, principalmente em cães de porte médio. Animais confinados a pequenos ambientes tenderam a apresentar miopia, enquanto os criados em locais amplos, a hipermetropia.

Pollet (1982) observou astigmatismo, com mais de 1,00 D, em $13,5 \%$, em olhos por ele examinados. Destes, $60,9 \%$ exibiam astigmatismo miópico simples. Murphy et al. (1992) detectaram astigmatismo de pelo menos $0,50 \mathrm{D}$, em $3,2 \%$ de olhos estudados, com variação de 0,50 a 3,00 D.

Pollet (1982) relatou a anisometropia de $0,50 \mathrm{D}$ como achado freqüente em cães, considerando-o dentro da faixa de normalidade. $\mathrm{O}$ autor trabalhou com 85 animais. Foram constatados $5,8 \%$ de animais com anisometropia de 1,00 a 2,00 D. Murphy et al. (1992) observaram, entre 240 animais avaliados, $16,0 \%$ de anisometropia, com, pelo menos, 0,50 D.

A raça Fila Brasileiro, tipicamente molossóide e genuinamente brasileira, caracteriza-se por indivíduos de grande porte, com aptidões para guarda, lides de gado e, ocasionalmente, caça de animais de grande porte (Valle e Monte, 1995). Para o desempenho de tais atividades, é mister que amonte carac- 
terísticas permissivas a uma boa acuidade visual tanto para campo próximo, quanto distante. Não há, todavia, estudos sobre condições que, objetivamente, integram e interferem com a acuidade visual na raça. Assim, objetivou-se determinar a refração ocular em cães da raça Fila Brasileiro, visando a se obterem dados quanto à condições ainda não notificadas, com vista ao estabelecimento de padrões próprios para a seleção de exemplares com os reais atributos para os quais a raça foi concebida.

\section{Material e método}

\section{Animais}

Foram utilizados 52 cães, da raça Fila Brasileiro, sendo 26 fêmeas, com idade entre um a dez anos, com peso corpóreo mínimo de 40 quilos, oriundos de canis credenciados pela Confederação Brasileira de Cinofilia (CBKC). Previamente à seleção os animais foram avaliados clinicamente e submetidos ao exame oftálmico rotineiro. Os cães foram selecionados para o estudo, quando isentos de alterações sistêmicas e oculares aparentes.

\section{Equipamentos}

A aferição da refração objetiva deu-se mediante o emprego de retinoscópio de faixa ${ }^{1}$ e régua de Parent $^{2}$ (Figura 1 A e B).

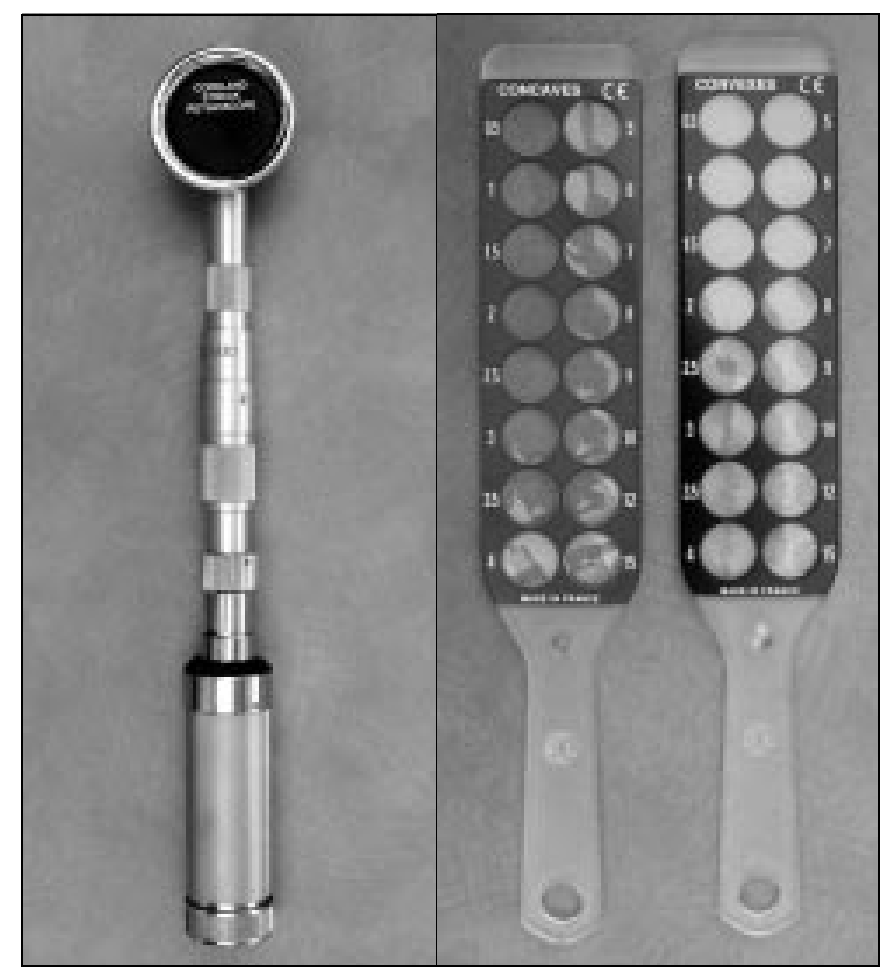

Figura 1: Em A, imagem fotográfica de retinoscópio de faixa Copeland. Em B, réguas de Parent para esquiascopia dotadas de lentes negativas (em vermelho) e lentes positivas (em preto).

\footnotetext{
${ }^{1}$ Retinoscópio de Copeland - Bausch-Lomb, SP, Brasil.

2 Paire de Règles à Skiascopie L2 Convex et Concave - Luneau Ophtalmologie, Chartres, France.
}

\section{Procedimentos}

Procedeu-se ao exame com o retinoscópio posicionado à distância de $66 \mathrm{~cm}$ do bulbo do olho e a régua a 2,00 centímetros (Davidson, 1997). Realizou-se avaliação do olho direito do paciente com o olho direito do examinador e, posteriormente, do olho esquerdo do paciente com o olho esquerdo do examinador, a fim de evitar paralaxe. Foram aferidos os meridianos horizontal e vertical (Pollet, 1982; Gaiddon et al., 1996).

O retinoscópio foi ajustado para emitir feixe de luz divergente. Inicialmente observou-se o reflexo de fundo, contra ou a favor, sem interposição da régua de esquiascopia. Ato contínuo, empregou-se a régua em incrementos de 0,50 D até se encontrar o ponto de neutralização. Do valor determinado, subtraiu-se a dioptria correspondente para obtenção do valor real (Nowak e Neumann, 1987; Gaiddon et al., 1996; Strubbe e Gelatt, 1999; Alves e Avakian, 2000), neste caso, de 1,50D.

\section{Análise estatística}

A análise estatística foi realizada valendo-se do teste nãoparamétrico de Wilcoxon, para comparação entre os olhos direito e esquerdo; Wilcoxon-Mann-Whitney, para comparação entre animais machos e fêmeas; e do coeficiente de correlação de Pearson para a verificação da existência, ou não, de associação significativa entre a idade e os dados da retinoscopia. O nível de determinação de significância adotado foi de $5 \%$. A análise estatística foi processada pelo software estatístico SPSS. ${ }^{3}$

\section{Resultados}

A retinoscopia em faixa foi realizada em 104 olhos de 52 cães da raça Fila Brasileiro. A contenção mecânica mostrou-se eficiente para a realização dos exames.

A refração média e respectivo desvio obtidos foram de $1,13 \pm 0,40 \mathrm{D}$, para o olho direito, e de $1,11 \pm 0,42 \mathrm{D}$ para o olho esquerdo, variando entre 0,50 a 2,00 D de hipermetropia. Não houve diferença significativa entre os olhos direito e esquerdo, quanto à refração aferida nos meridianos horizontal, vertical e média, a um nível de significância de 5\% (Tabela 1).

Apesar de as medidas diretas apresentarem uma precisão de 0,50 D, o erro de refração foi estimado em 0,25 D. Dos 104 olhos, 24,04\% apresentaram hipermetropia de 0,50 a $0,75 \mathrm{D}$; $40,38 \%$ de 1,00 a 1,25 D; $31,43 \%$ de 1,50 a 1,75 D e $3,85 \%$ de 2,00 D.

Dos 104 olhos avaliados, 17 (16,35\%) apresentaram baixo grau de astigmatismo hipermetrópico composto, que correspondeu a 0,50 D. Desses, em dois animais a manifestação foi bilateral e em 13, unilateral.

A anisometropia hipermetrópica composta constituiu achado freqüente, tendo sido encontrada em 22 animais (42,31\%). Destes, em 21 a diferença foi $=0,50 \mathrm{D}$ e em um foi de 0,75 D.

Não se observou diferença significativa $(p>0,05)$ na refração dos meridianos horizontal, vertical e média do olho direito e esquerdo entre machos e fêmeas (Tabela 2).

Observou-se não ter havido associação significativa entre a idade e a refração nos meridianos horizontal, vertical e média ( $\mathrm{r} I \leq 0,6)$, o que pode ser exemplificado, relativamente à refração média de cada olho, na Figura 2.

\footnotetext{
${ }^{3}$ Statistical Package for the Social Sciences, Version 10.0.1, 1999. SPSS Inc. Illinois, USA.
} 
Tabela 1: Total de olhos examinados, médias, desvios-padrão e valores mínimos e máximos da refração obtida à retinoscopia com luz em faixa dos bulbos dos olhos direito (OD) e esquerdo $(\mathrm{OE})$ de 52 cães da raça Fila Brasileiro

\begin{tabular}{ccccc}
\hline Refração & $\mathbf{n}$ & Meridiano horizontal (D) & Meridiano vertical (D) & Média (D) \\
\hline Olho direito & & $1,13 \pm 0,42$ & $1,13 \pm 0,41$ & $1,13 \pm 0,40$ \\
Variação (mín; máx) & \multirow{2}{*}{52} & $(0,50 ; 2,00)$ & $(0,50 ; 2,00)$ & $(0,50 ; 2,00)$ \\
Olho esquerdo & \multirow{2}{*}{52} & $1,10 \pm 0,43$ & $1,10 \pm 0,42$ & $1,11 \pm 0,42$ \\
Variação (mín; máx) & & $(0,50 ; 2,00)$ & $(0,50 ; 2,00)$ & $(0,50 ; 2,00)$ \\
Média & \multirow{2}{*}{104} & $1,11 \pm 0,43$ & $1,12 \pm 0,41$ & $1,12 \pm 0,41$ \\
Variação (mín; máx) & & $(0,50 ; 2,00)$ & $(0,50 ; 2,00)$ & $(0,50 ; 2,00)$ \\
Wilcoxon & \multirow{2}{*}{ p-valor } & 0,467 & 0,346 & 0,515 \\
OD x OE & & & & \\
\hline
\end{tabular}

$\mathrm{n}=$ número de olhos examinados; $\mathrm{D}=$ dioptrias; $\mathrm{OD} \times \mathrm{OE}=\mathrm{compa}$ ração das aferições nos olhos direito (OD) e esquerdo (OE), com base nos valores medidos em cada indivíduo; $p>0,05$ não significativo a $5 \%$.

Tabela 2: Total de olhos examinados, médias, desvios-padrão e valores mínimos e máximos, segundo o sexo, da refração obtida à retinoscopia com luz em faixa dos bulbos dos olhos direito (OD) e esquerdo (OE) de 52 cães da raça Fila Brasileiro

\begin{tabular}{|c|c|c|c|c|}
\hline Refração & $\mathbf{n}$ & Meridiano horizontal (D) & Meridiano vertical (D) & Média (D) \\
\hline $\begin{array}{c}\text { Machos } \\
\text { OD } \\
\text { Variação (mín; máx) }\end{array}$ & 26 & $\begin{array}{c}1,12 \pm 0,38 \\
(0,50 ; 1,50)\end{array}$ & $\begin{array}{c}1,08 \pm 0,34 \\
(0,50 ; 1,50)\end{array}$ & $\begin{array}{c}1,10 \pm 0,35 \\
(0,50 ; 1,50)\end{array}$ \\
\hline $\begin{array}{c}\text { OE } \\
\text { Variação (mín; máx) }\end{array}$ & 26 & $\begin{array}{c}1,08 \pm 0,39 \\
(0,50 ; 1,50)\end{array}$ & $\begin{array}{c}1,06 \pm 0,36 \\
(0,50 ; 1,50)\end{array}$ & $\begin{array}{c}1,09 \pm 0,35 \\
(0,50 ; 1,50)\end{array}$ \\
\hline $\begin{array}{c}\text { Ambos } \\
\text { Variação (mín; máx) }\end{array}$ & 52 & $\begin{array}{c}1,10 \pm 0,38 \\
(0,50 ; 1,50)\end{array}$ & $\begin{array}{c}1,07 \pm 0,34 \\
(0,50 ; 1,50)\end{array}$ & $\begin{array}{c}1,09 \pm 0,35 \\
(0,50 ; 1,50)\end{array}$ \\
\hline $\begin{array}{c}\text { Fêmeas } \\
\text { OD } \\
\text { Variação (mín; máx) }\end{array}$ & 26 & $\begin{array}{c}1,13 \pm 0,46 \\
(0,50 ; 2,00)\end{array}$ & $\begin{array}{c}1,19 \pm 0,47 \\
(0,50 ; 2,00)\end{array}$ & $\begin{array}{c}1,16 \pm 0,46 \\
(0,50 ; 2,00)\end{array}$ \\
\hline $\begin{array}{c}\text { OE } \\
\text { Variação (mín; máx) }\end{array}$ & 26 & $\begin{array}{c}1,12 \pm 0,50 \\
(0,50 ; 2,00)\end{array}$ & $\begin{array}{c}1,13 \pm 0,48 \\
(0,50 ; 2,00)\end{array}$ & $\begin{array}{c}1,13 \pm 0,48 \\
(0,50 ; 2,00)\end{array}$ \\
\hline $\begin{array}{c}\text { Ambos } \\
\text { Variação (mín; máx) }\end{array}$ & 52 & $\begin{array}{c}1,13 \pm 0,47 \\
(0,50 ; 2,00)\end{array}$ & $\begin{array}{c}1,16 \pm 0,47 \\
(0,50 ; 2,00)\end{array}$ & $\begin{array}{c}1,14 \pm 0,46 \\
(0,50 ; 2,00)\end{array}$ \\
\hline $\begin{array}{c}\text { W-M-W } / \text { Grupo = } \\
\text { sexo } \\
\text { OD } \\
\text { OE }\end{array}$ & $\begin{array}{l}p- \\
\text { valor } \\
p- \\
\text { valor }\end{array}$ & $\begin{array}{l}0,822 \\
0,893\end{array}$ & $\begin{array}{l}0,274 \\
0,752\end{array}$ & $\begin{array}{l}0,457 \\
0,977\end{array}$ \\
\hline
\end{tabular}

$\mathrm{n}=$ número de olhos examinados: $\mathrm{D}=$ dioptrias; $\mathrm{W}-\mathrm{M}-\mathrm{W}=$ WilcoxonMann-Whitney; grupo - sexo OD = comparação entre as medidas de machos e fêmeas para os olhos direitos; grupo - sexo OE = comparação entre as medidas de machos e fêmeas para os olhos esquerdos: p>0,05 não significativo a $5 \%$, na comparação dos grupos.

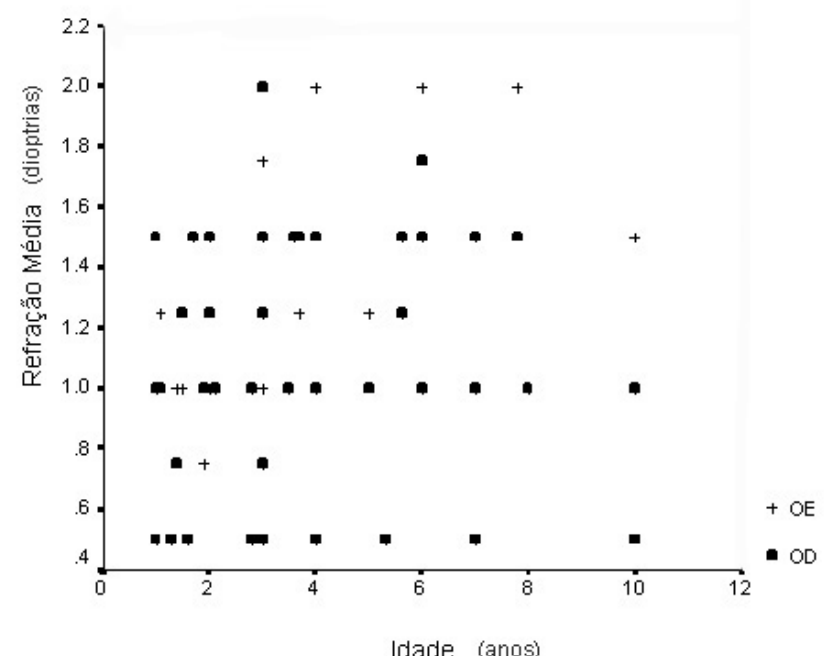

Figura 2: Representação gráfica da relação entre a refração média (em dioptrias) e a idade (em anos), para os bulbos dos olhos esquerdo (OE) e direito (OD) de 52 cães da raça Fila Brasileiro submetidos a retinoscopia com luz em faixa.

\section{Discussão}

Em medicina, após a consecução da refração objetiva, confirma-se o erro refracional mediante a realização de exame subjetivo (Netto, 2000). Porém, em veterinária, a refração subjetiva não é realizada. Desse modo, resultados de $\pm 0,50 \mathrm{D}$ a partir do real estado de refração do animal são considerados adequados (Davidson, 1997).

No presente estudo observou-se baixo grau de hipermetropia em todos os animais $(1,13 \pm 0,40 \mathrm{D}$ para o olho direito e $1,11 \pm 0,42 \mathrm{D}$ para o olho esquerdo, variando de 0,50 a 2,00 D). Especula-se que, similarmente ao que foi observado por Murphy et al. (1992), em cães da raça Pastor Alemão, utilizados como guias para cegos, a seleção, com relação às funções da raça, tenha excluído animais com problemas de desempenho.

Detectou-se tendência familiar à ocorrência de ametropias em raças como Rottweiler, Schnauzer Miniatura, Pastor Alemão (Murphy et al., 1992; Mutti et al., 1999) e Retriever do 
Labrador (Nelson et al., 1996; Mutti et al., 1999). Tal não foi estabelecido na pesquisa ora conduzida, pois os resultados revelaram constante hipermetropia, não permitindo a correlação de dados.

Em contraste com os achados de Murphy et al. (1992) e Mutti et al. (1999), que encontraram inter-relação estatisticamente significativa quanto à idade e erro de refração, especialmente em animais idosos, o presente estudo não a evidenciou. Aventa-se, assim como Pollet (1982) o fizera, que, em cães, diferenças raciais sejam mais relevantes que diferenças etárias.

Analogamente aos resultados de Mutti et al. (1999), astigmatismo de baixa magnitude $(0,50 \mathrm{D})$ foi detectado em $16,35 \%$ dos olhos, e classificado, em relação ao erro refrativo, como hipermetrópico composto. Esses resultados se contrapõem com os de Pollet (1982), em que o grau de astigmatismo detectado foi mais elevado ( = 1,00 D) dandose em $14 \%$ dos olhos examinados, sendo o miópico simples o mais freqüente.
Os achados relativos ao leve e freqüente grau de anisometropia assemelham-se aos obtidos por Pollet (1982). Em seres humanos, pequenas anisometrias são comumente vistas, porém não causam transtornos à visão binocular. Todavia, graus acima de 3,0 D de anisometropia embargam a fusão das imagens retinianas (Giovedi Filho e Alves, 2000). No estudo ora conduzido, anisometropia hipermetrópica composta de 0,50 D foi considerada normal.

\section{Conclusões}

A base dos estudos concebidos visando a se investigarem os valores da refração ocular por retinoscopia em faixa, em cães da raça Fila Brasileiro, permite admitir que:

- a técnica de exame fornece resultados fidedignos e é bem tolerada pelos pacientes;

- o valor médio da refração ocular do Fila Brasileiro é de $1,12 \pm 0,41 \mathrm{D}$

- a raça Fila Brasileiro apresenta, em média, grau leve de hipermetropia.

\section{Agradecimentos}

- CAPES / PICDT / UFF - pelo apoio ao desenvolvimento da pesquisa.

- Aos proprietários dos canis Cachoeira, Clamofá, Entrefolhas, Pedra de Guaratiba, Quizanga, e senhor "Betinho", que possibilitaram a realização deste estudo.

- Ao médico-veterinário André Saramago, pela colaboração na fase experimental da pesquisa.

\section{Referências}

ALVES, A. A. A prática da retinoscopia à luz em faixa. In: Refração. 3. ed. Rio de Janeiro: Cultura Médica, 1999. p. 168-182.

ALVES, M. R.; AVAKIAN, A. Testes objetivos e subjetivos do exame de refração. In: URAS, R. Coleção de manuais básicos CBO: óptica e refração ocular. São Paulo: Cultura Médica, 2000. p. 75-96.

DAVIDSON, M. G. et al. Refractive state of aphakic and pseudophakic eyes of dogs. Am. J. Vet. Res., Schaumburg, v. 54, n. 1, p. 174-177, 1993.

DAVIDSON, M. G. Clinical retinoscopy for the veterinary ophthalmologist. Vet. Comp. Ophthalmol., Irvine, v. 7, n. 2, p. 128-137, 1997.

GAIDDON, J. et al. Use of biometry and keratometry for determining optimal power for intraocular lens implants in dogs. Am. J. Vet. Res., Schaumburg, v. 52, n. 5, p. 781-783, 1991.

GAIDDON, J.; BOUHANA, N.; LALLEMENT, P. E. Refraction by retinoscopy of normal, aphakic, and pseudophakic canine eyes: advantage of a 41-diopter intraocular lens? Vet. Comp. Ophthalmol., Irvine, v. 6, n. 2, p. 121-124, 1996.

GAIDDON J. A.; LALLEMENT, P. E.; PEIFFER, Jr, R. L. Implantation of a foldable intraocular lens in dogs. J. Vet. Med. Assoc., Schaumburg, v. 216, n. 6, p. 875-877, 2000.

GILGER, B. C.; DAVIDSON, M. G.; HOWARD, P. B. Keratometry, ultrasonic biometry, and prediction of intraocular lens power in the feline eye. Am. J. Vet. Res., Schaumburg, v. 59, n. 2, p. 131-134, 1998.

GIOVEDI FILHO, R.; ALVES, M. R. Anisometropia. In: URAS, R. Coleção de manuais básicos $C B O$ : óptica e refração ocular. São Paulo: Cultura Médica, 2000. p. 67-74.

MILLER, P.; MURPHY, C. J. Vision in dogs. J. Am. Vet. Med. Assoc., Schaumburg, v. 207, p. 1623-1633, 1995.

MURPHY, C. J. et al. Myopia and refractive error in dogs. Invest. Ophthalmol. Vis. Sci., Brookline, v. 33, n. 8, p. 2459-2463, 1992.
MURPHY, C. J. et al. Effect of optical defocus on visual acuity in dogs. Am. J. Vet. Res., Schaumburg, v. 58, n. 4, p. 414-417, 1997.

MUTTI, D. O.; ZADNIK, K.; MURPHY, C. J. Naturally occurring vitreous chamber-based myopia in the Labrador retriever. Invest. Ophthalmol. Vis. Sci., Brookline, v. 40, n. 7, p. 1577-1584, 1999.

NELSON, M. J. et al. A naturally occuring model of myopia in the labrador retriever. Invest. Ophthalmol. Vis. Sci., Brookline, suppl:1485, 1996. (Abstract B 388).

NETTO, A. L. Emetropias e ametropias: testes subjetivos e objetivos. In: URAS, R. Coleção de manuais básicos $C B O$. Óptica e refração ocular. São Paulo: Cultura Médica, 2000. p. 51-66.

NEUMANN, W. Biometrie am hundeauge. Kleintierpraxis, v. 33, n. 4, p. 113-152, 1988.

NOWAK, M. R.; NEUMANN, W. Refraktion des hundeauges. Klin. Monatsbl. Augenheilkd., Stuttgart, v. 191, p. 81-83, 1987.

OFRI, R. Optics and physiology of vision. In: GELATT, K. N. Veterinary ophthalmology. 3. ed. Baltimore: Lippincott Williams and Wilkins, 1999. p. 183-218.

POLLET, L. Refraction of normal and aphakic canine eyes. J. Am. Anim. Hosp. Assoc., Lakewood, v. 18, p. 323-326, 1982.

ROSOLEN, S. G. et. al. Refractive corneal surgery on dogs: preliminary results of keratomileusis using a 193 nanometer eximer laser. Vet. Comp. Ophthalmol., Irvine, v. 10, n. 5, p. 18-24, 1995.

STRUBBE, D. T.; GELATT, K. N. Ophthalmic examination and diagnostic procedures. In: GELATT, K. N. Veterinary ophthalmology. 3. ed. Baltimore: Lippincott Williams and Wilkins, 1999. p. 427-466.

VALLE, P. J.; MONTE, E. O grande livro do Fila brasileiro. 2. ed. São Paulo: Nobel e Brazels Wallace, 1995. p. 295-312.

WYMAN, M.; DONOVAN, E. F. The ocular fundus of the normal dog. J. Am. Vet. Med. Assoc., Schaumburg, v. 147, n. 1, p. 17-26, 1965.

ASSOCIAÇÃOBRASILEIRA DE NORMAS TÉCNICAS. NBR 6023: informação e documentação: referências: elaboração. Rio de Janeiro, 2002. 